\title{
QUEEN'S
UNIVERSITY
BELFAST
}

\section{Numerical study of neutron beam divergence in a beam-fusion scenario employing laser driven ions}

Alejo, A., Green, A., Ahmed, H., Robinson, A. P. L., Cerchez, M., Clarke, R., Doria, D., Dorkings, S., Fernandez, J., McKenna, P., Mirfayzi, S. R., Naughton, K., Neely, D., Norreys, P., Peth, C., Powell, H., Ruiz, J. A., Swain, J., Willi, O., ... Kar, S. (2016). Numerical study of neutron beam divergence in a beam-fusion scenario employing laser driven ions. Nuclear Instruments and Methods in Physics Research Section A: Accelerators, Spectrometers, Detectors and Associated Equipment, 829, 176-180. https://doi.org/10.1016/j.nima.2016.05.057 Published in:

Nuclear Instruments and Methods in Physics Research Section A: Accelerators, Spectrometers, Detectors and Associated Equipment

Document Version:

Peer reviewed version

Queen's University Belfast - Research Portal:

Link to publication record in Queen's University Belfast Research Portal

\section{Publisher rights}

(C) 2016 Elsevier B.V. This manuscript version is made available under the CC-BY-NC-ND 4.0 license

http://creativecommons.org/licenses/by-nc-nd/4.0/,which permits distribution and reproduction for non-commercial purposes, provided the author and source are cited.

\section{General rights}

Copyright for the publications made accessible via the Queen's University Belfast Research Portal is retained by the author(s) and / or other copyright owners and it is a condition of accessing these publications that users recognise and abide by the legal requirements associated with these rights.

Take down policy

The Research Portal is Queen's institutional repository that provides access to Queen's research output. Every effort has been made to ensure that content in the Research Portal does not infringe any person's rights, or applicable UK laws. If you discover content in the Research Portal that you believe breaches copyright or violates any law, please contact openaccess@qub.ac.uk. 


\title{
Numerical study of neutron beam divergence in a beam-fusion scenario employing laser driven ions
}

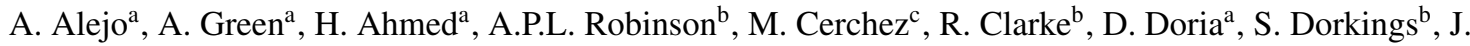 \\ Fernandez $^{\mathrm{b}}$, P. McKenna ${ }^{\mathrm{d}}$, S.R. Mirfayzi ${ }^{\mathrm{a}}$, K. Naughton ${ }^{\mathrm{a}}$, D. Neely ${ }^{\mathrm{b}}$, P. Norreys ${ }^{\mathrm{b}, 1}$, C. Peth $^{\mathrm{c}}$, H. Powell $^{\mathrm{d}}$, J.A. Ruiz $^{\mathrm{e}}$, \\ J. Swain ${ }^{\mathrm{f}}$, O. Willic, M. Borghesi ${ }^{\mathrm{a}}, \mathrm{S}$. Kar ${ }^{\mathrm{a}, *}$ \\ ${ }^{a}$ Centre for Plasma Physics, School of Mathematics and Physics, Queen's University Belfast, BT7 1NN, UK \\ ${ }^{b}$ Central Laser Facility, Rutherford Appleton Laboratory, Didcot, Oxfordshire, OX11 OQX, UK \\ ${ }^{c}$ Institut für Laser-und Plasmaphysik, Heinrich-Heine-Universität, Düsseldorf, 40225, Germany \\ ${ }^{d}$ Department of Physics, SUPA, University of Strathclyde, Glasgow, G4 ONG, UK \\ ${ }^{e}$ Colegio Los Naranjos, Fuenlabrada, Madrid, 28941, Spain \\ ${ }^{f}$ Rudolf Peierls Centre for Theoretical Physics, University of Oxford, Oxford, OX1 3NP, UK
}

\begin{abstract}
The most established route to create a laser-based neutron source is by employing laser accelerated, low atomic number ions in fusion reactions. In addition to benefiting from the high reaction cross-sections at moderate energies of the projectile ions, the anisotropy in the neutron emission is another important feature of beam-fusion reactions. Using a simple numerical model based on neutron generation in a pitcher-catcher scenario, anisotropy in the neutron emission was studied for the deuterium-deuterium fusion reaction. Simulation results are consistent with the narrowdivergence $\left(\sim 70^{\circ}\right.$ full width at half maximum) neutron beam recently obtained from an experiment employing multi$\mathrm{MeV}$ deuteron beams of narrow divergence (upto $30^{\circ} \mathrm{FWHM}$ depending on the ion energy) accelerated by a subpetawatt laser pulse from thin deuterated plastic foils via the Target Normal Sheath Acceleration mechanism. By varying the input ion beam parameters, simulations show that a further improvement in the neutron beam directionality (i.e. reduction in the beam divergence) can be obtained by increasing the projectile ion beam temperature and cut-off energy, as expected from the interactions with higher power lasers at upcoming facilities.
\end{abstract}

Keywords: laser, neutron, beam fusion

\section{Introduction}

Fast neutron sources driven by high-power lasers have gained substantial interest over the last decades for a range of potential applications in medicine [1], security [2,3], material science [4] and high energy density physics research [5]. Furthermore, deploying compact moderators closely coupled to laser-driven fast neutrons sources would allow the development of intense sources of thermal and epithermal neutrons, which would extend the range of applicability of laser-based sources. With the rapid progress in laser technology, aiming towards developing higher repetition rate lasers of higher powers, laser-driven neutron sources can, in principle, complement the research activities currently pursued at

\footnotetext{
${ }^{*}$ Corresponding author.
}

Email address: s.kar@qub.ac.uk conventional accelerator-driven spallation sources. Although these large scale facilities produce substantially higher fast neutron fluxes, a key interest for laser-driven neutron sources lies in the neutron burst duration, which is substantially shorter than that produced at spallation facilities.

With the current laser systems, neutron yields up to of the order of $10^{10}$ neutrons/shot have been shown experimentally $([6,7]$ and references therein), by employing laser driven ions to generate neutrons from secondary catcher targets via beam-fusion reactions. In addition to the advantage of the ultra-short pulse duration, directionality/anisotropicity in the neutron emission is another important characteristic resulting from the beam-fusion reactions. The total neutron yield from a fusion reaction scales with the product of fusing ion densities and cross-section $\sigma$, which, for the most common reactions, reaches high values for centre-of- 
mass energy in the $\mathrm{MeV}-10 \mathrm{~s}$ of $\mathrm{MeV}$ range [8]. In a 8 pitcher-catcher scenario, where neutrons are produced by bombarding the laser-driven ions on a suitable converter target, anisotropy arises from the nuclear reaction kinematics, which strongly depends on the atomic mass of the fusing nuclei and velocity of the projectile ions [6]. In addition, the strong angular dependence of the differential cross-section for light nuclei reactions helps producing neutron fluxes strongly peaked along the incident ion-beam direction, even while using moderate energy (10s of $\mathrm{MeV}$ ) ions as accelerated at currently available laser intensities [9]. In this context, fusion reactions based on low atomic mass nuclei, such as ${ }^{7} \mathrm{Li}(\mathrm{p}, \mathrm{n}){ }^{7} \mathrm{Be},{ }^{9} \mathrm{Be}(\mathrm{p}, \mathrm{n}){ }^{9} \mathrm{~B},{ }^{13} \mathrm{C}(\mathrm{p}, \mathrm{n}){ }^{13} \mathrm{~N}$, $\mathrm{d}(\mathrm{d}, \mathrm{n}){ }^{3} \mathrm{He}, \mathrm{T}(\mathrm{d}, \mathrm{n}){ }^{4} \mathrm{He},{ }^{7} \mathrm{Li}(\mathrm{d}, \mathrm{xn}),{ }^{7} \mathrm{Be}(\mathrm{d}, \mathrm{xn})$, are particularly relevant, which would not only allow obtaining higher neutron yield, but higher peak flux by producing a narrow cone beam of neutrons. A highly beamed neutron flux would be extremely helpful towards improving transport capabilities as well as efficient moderation of the neutrons to thermal and epithermal energies by using compact, closely-coupled, directional moderators.

Anisotropic emission of the neutron beam is starting to be realized in experiments. In addition to the anisotropicity intrinsic to the beam-fusion, as discussed above, the neutron beam divergence from a typical laser-driven pitcher-catcher source also depends strongly on the divergence of the projectile ions - the final neutron beam divergence from the catcher will be a convolution of the divergence of the input ion beam and the neutron beam divergence expected for a collimated beam of ions.

In this paper we show a simple model for simulating the neutron production from light nuclei reactions in a pitcher-catcher scenario, and to study the effect of ion beam parameters (divergence and spec- 102 trum) on the neutron generation. The neutron beam 103 divergence estimated by our model from $\mathrm{d}(\mathrm{d}, \mathrm{n})^{3} \mathrm{He}$ re- ${ }_{104}$ action in a beam-fusion scenario, while using laser- 105 driven deuterium beam produced via the Target Normal 106 Sheath Acceleration (TNSA) mechanism[9], compares 107 well with the data obtained from a recent experiment 108 [6]. A systematic study show that the neutron beam di- 109 vergence can be reduced significantly (to a few tens of 110 degrees) by increasing the input ion beam temperature, 111 which, according to the current understanding of the 112 TNSA mechanism, is achievable using the intense lasers 113 that will be available at the upcoming facilities[10, 11]. ${ }_{114}$

\section{Simulation design and method}

Alternative to the usual Monte-Carlo approach [12, 13] to simulate neutron generation in a beam-fusion scenario, the model described in this paper (as discussed below) takes advantage of the tabulated angularlyresolved neutron yield, that can be found in the literature, obtained for a mono-energetic, pencil beam of ions impinging onto a catcher at normal incidence. The main interaction that is taken into account in our simple model is the effect of a multi-energy, divergent beam of ions, as typically produced by the TNSA mechanism, towards the angular distribution of emitted neutrons from a secondary catcher target. The schematic of the setup used in our model is shown in Fig. 1.

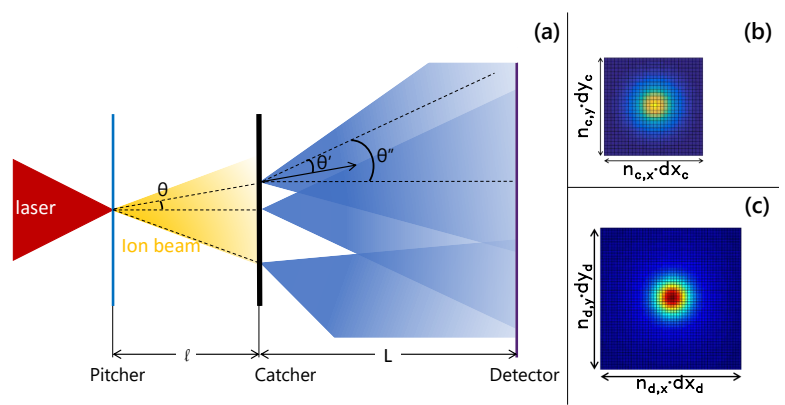

Figure 1: (a) Schematic of the neutron generation process in the pitcher-catcher scheme. An ion beam is generated by the interaction of the laser with the target (pitcher), which then reaches the secondary converter (catcher), leading to the neutron emission. (b) and (c) depict the grids representing the catcher and the detector, respectively.

The input for the projectile ion beam in our model is the angularly-resolved ion spectra. This information can either be obtained numerically, by performing multi-dimensional PIC simulations of the laser-foil interaction, or experimentally, by using for example angularly distributed high-resolution Thomson Parabola Spectrometers (TPS) [14]. The angularly resolved ion spectra can be represented by a function $\frac{d^{2} N_{i o n}}{d E d \Omega}(E, \theta, \varphi)$, where $E$ is the ion energy, $(\theta, \varphi)$ are the angles defining the direction of a given beamlet of ion, and $\Omega$ stands for solid angle. For simplicity, one may assume the ion beam produced by the laser-foil interaction to be cylindrically symmetrical about $\theta$.

The catcher in the model was designed as a two dimensional matrix $\left(n_{c, x} \times n_{c, y}\right.$ cells), where the grid size $\left(d x_{c} \times d y_{c}\right)$ can be chosen depending on the desired resolution and accuracy, being $d x_{c}=d y_{c}=200 \mu \mathrm{m}$ the resolution for the simulations here shown. The spectrum of ions arriving at each grid point on the catcher $\left(d N_{\text {ion, }(x, y)} / d E\right)$ is calculated from the input ion spectrum 
to the code (as mentioned above) for a given pitcher-tocatcher distance $(l)$. In order to obtain the neutron flux distribution across a plane parallel to the catcher, the detector in the code was modelled as a two dimensional array of $n_{d, x} \times n_{d, y}$ cells of size $d x_{d} \times d y_{d}$. This detector configuration mimics the response of CR39 nuclear track detectors typically used in neutron generation experiments [6? , 15], allowing for a direct comparison between the simulations and the experimental data.

Neutron generation from each grid point of the catcher was calculated by using the tabulated data for the angularly-resolved neutron yield, that can either be found in the literature, or be obtained by running Monte Carlo simulations $[12,13]$ for different ion energies. In this paper we used the tabulated data for $d(d, n)^{3} \mathrm{He}$ reaction provided by Davis et al. [12], which was one of the main reactions producing neutron in the experiment reported in [6]. The $\mathrm{d}(\mathrm{d}, \mathrm{n})^{3} \mathrm{He}$ reaction is also an efficient fusion reaction for moderate-energy deuterons, which is suitable for studying the effect of the input ion beam spectrum and divergence on the neutron beam divergence. The tabulated neutron yield per incident ion, given in Ref. [12], along different angles of neutron emission and for different projectile ion energies were interpolated to obtain a function $Y_{n}\left(E, \theta^{\prime}\right)$, where $\theta^{\prime}$ is the neutron emission angle with respect to the incident ion beam. Using this function, the neutron flux at a given pixel of the detector $\left(F_{n,\left(x_{d}, y_{d}\right)}=N_{n,\left(x_{d}, y_{d}\right)} / d x_{d} d y_{d}\right)$ is calculated as the sum of the fluxes reaching that pixel, generated at each point on the catcher. This can be expressed mathematically as

$$
N_{n,\left(x_{d}, y_{d}\right)}=\sum_{\left(x_{c}, y_{c}\right)} \sum_{E} Y_{n}\left(E, \theta^{\prime \prime}\right) \cdot N_{\text {ion, }\left(x_{c}, y_{c}\right)}(E)
$$

where, $\theta^{\prime \prime}=\tan ^{-1}\left(\sqrt{\left(x_{d}-x_{c}\right)^{2}+\left(y_{d}-y_{c}\right)^{2}} / L\right)$ and $L$ is the catcher-to-detector distance.

\section{Results}

In order to study the beamed neutron emission ob- 184 served in our experiment [6] employing the petawatt 185 arm of the VULCAN laser at Rutherford Appleton Lab- 186 oratory (RAL), STFC, UK[16], we used the experimen- 187 tally measured deuteron spectrum as the input to our 188 model. The ion beams in the experiment were pro- 189 duced by irradiating $10 \mu \mathrm{m}$-thick deuterated plastic foils 190 with a p-polarised laser pulse of $\sim 200 \mathrm{~J}$ energy and 191 $\sim 750 \mathrm{fs}$ duration, focussed down to a spot of $\sim 6 \mu \mathrm{m} \quad 192$ (FWHM) on the target, reaching a peak intensity in ex- 193 cess of $10^{20} \mathrm{~W} \mathrm{~cm}^{-2}$. The ion beam spectrum was di- 194 agnosed along different emission angles $\left(-8^{\circ}, 0^{\circ}, 21^{\circ}{ }_{195}\right.$

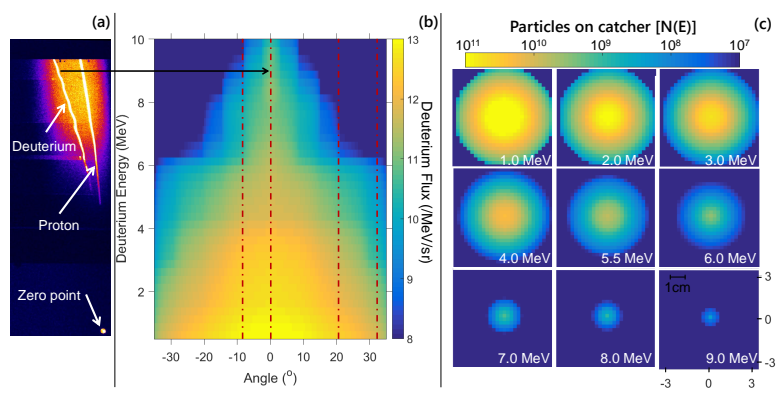

Figure 2: (a) A typical raw data obtained by a TPS diagnosing ion spectrum along the target normal direction. (b) Angularly resolved deuteron spectrum reconstructed from the data obtained by four TPS measurements along different angles (as shown by the dashed line) with respect to the target normal direction. (c) Spatial profiles of deuteron beam for different energies at the catcher plane.

and $32^{\circ}$ ) with respect to the target normal by employing Thomson Parabola Spectrometers (TPS)[14]. Due to the limitation of TPS in retrieving the spectrum of overlapping species, a differential filtering technique [17] was implemented to discriminate the deuterium ions from the overlapping species with equal charge-to-mass ratio, such as $C^{6+}$ and $O^{8+}$ originated from the target and hydrocarbon contaminant layers. A typical raw data obtained along the target normal direction is shown in Fig. 2(a). A comparison between on-axis proton and deuteron spectra obtained from the TPS data is shown in Ref. [6]. The deuteron spectra obtained from the different TPS were used to reconstruct the full beam profile, as shown in Fig. 2(b), while assuming an axissymmetrical beam profile. The data shows a divergent ( $\sim 30^{\circ} \mathrm{FWHM}, \sim 60^{\circ}$ full cone) beam of deuterons with an exponential spectrum, with the highest energies produced along the target normal direction with a narrow ( $\sim 15^{\circ}$ full cone) beam divergence, as expected from the TNSA mechanism for such laser and target parameters [18].

The angularly-resolved deuteron spectra shown in Fig. 2(c) was used in our code to simulate the neutron generation in the catcher placed at a distance of $l=5 \mathrm{~mm}$ from the ion source (which represents the point of laser interaction with the pitcher target). The flux distribution of the deuterons of different energies at the front surface of the catcher are shown in Fig. 2(c), which was obtained by using the beam profile shown in Fig. 2(b).

Despite of the moderate energies of the ions and the broad emission angle produced in the experiment, the simulation shows a directional beam-like emission of neutrons from the catcher target, as shown in Fig. 3 (showing neutron flux distribution across the detector 
plane placed at a distance $L=15 \mathrm{~mm}$ from the catcher), with a Full Width at Half Maximum (FWHM) divergence of $\sim 62^{\circ}$ and maximum flux along the ion beam axis. The simulated neutron beam profile is similar to that obtained from the experiment (FWHM of $(70 \pm 10)^{\circ}$ [6]). Since the simulated neutron beam profile was obtained by considering only the $d(d, n)^{3} \mathrm{He}$ reaction, the residual difference between the simulated and experimental neutron beam profile is most likely due to a range of additional nuclear reactions taking place in the catcher in the latter case. As discussed in Ref. [6], due to the higher flux and higher energy protons produced from the pitcher target (as can be seen in Fig. 2(a)), the proton-induced deuteron breakup reaction $\left(d(p, n+p){ }^{1} H\right)$ contributes significantly towards the total neutron yield. Due to the reaction kinematics, this nuclear reaction is expected to produce a narrow neutron beam divergence, similar to that obtained for the $d(d, n)^{3} \mathrm{He}$ reaction. However, a detailed simulation for the $d(p, n+p)^{1} H$ case could not be carried out due to the insufficient reaction cross-section available in the literature.

In order to study the effect of the projectile ion beam ${ }^{217}$ parameters on the neutron beam divergence, we car- ${ }^{218}$ ried out a set of simulations by varying the input spec- ${ }^{219}$ trum of the ions, as expected to be produced by TNSA ${ }^{220}$ mechanism at different laser intensities. The ion tem- ${ }^{221}$ perature and the cut-off ion energy in the TNSA mech- ${ }^{222}$ anism scale with the temperature of the hot electrons ${ }^{223}$

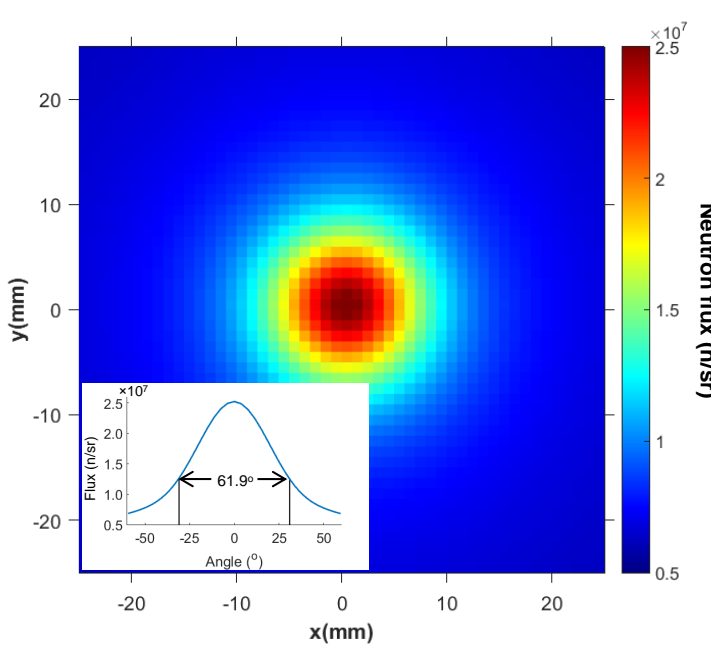

Figure 3: Simulated neutron beam reaching a flat detector in front of the catcher. Inset shows the lineout of the neutron beam profile across the detector, which also represents the emission angle of neutrons with respect to the ion beam axis. The divergence of the neutron beam (FWHM) is $\sim 62^{\circ}$. produced by the interaction, which broadly scales as $\sqrt{I_{L} \lambda^{2}}[9,19,20,21]$, where $I_{L}$ and $\lambda$ stand, respectively, for the intensity and the wavelength of the incident laser. The divergence of the ions produced by the TNSA mechanism also varies within the beam depending on its energy [18] - ions with higher energy are emitted with a lower divergence. Assuming a flat-top flux profile within the ion beam divergence, and the following divergence profile as a function of ion energy (as reported for $\sim$ ps lasers in Ref. [18], which closely matches with the observed divergence shown in Fig. 2(b)),

$$
\theta_{D}\left(E, E_{\text {max }}\right)=\left\{\begin{array}{ll}
62 & E<E_{\max } / 2 \\
107.4-90.9 \cdot \frac{E}{E_{\max }} & E \geq E_{\max } / 2
\end{array},\right.
$$

we modelled an input TNSA beam profile for our simulations as a function of laser intensity, as given by

$$
\frac{d^{2} N_{\text {ion }}}{d E d \Omega}=\left.\frac{d N_{\text {ion }}}{d E}\right|_{\substack{E<E_{\max } \\ \theta<\theta_{D}}} \propto \exp \left(-\frac{E}{k_{B} T\left(I_{L}\right)}\right)
$$

A set of simulations were carried out by varying the ion beam temperature $k_{B} T\left(I_{L}\right)$. The cut-off energy for the deuterons as a function of laser intensities was assumed as $E_{\max }\left(I_{L}\right) \propto 10^{-9} \sqrt{I_{L}} \mathrm{MeV}$, where the proportionality constant was calculated using the maximum deuteron energy obtained in our experiment, shown in Fig. 2(b).

The FWHM divergence of the neutron beam obtained from the simulations is shown in Fig. 4. One can see how the neutron beam divergence reduces significantly with an increase in the ion beam temperature. While a nearly isotropic emission for low ion temperatures is produced, the neutron beam divergence can be reduced below $50^{\circ}$ using higher power lasers than that used in our experiment. Intense lasers will produce ions at higher energies, which will provide two-fold enhancement to the on-axis neutron flux - (1) neutron yield per incident ion will increase significantly due to their deeper penetration into the catcher, (2) higher anisotropicity due to differential cross-section and kinematics (see Eq. 2 in Ref. [6]). An alternative approach for increasing the flux and energy of ions other than protons, which are preferentially accelerated by the TNSA mechanism, would be to use some special technique to eliminate the hydrogen contamination layer at the rear side of the pitcher target, such as depositing a layer of heavy water contamination for enhancing the deuteron acceleration [22].

The rate of decrease in the neutron beam divergence slows down towards the higher temperatures, as visible in Fig. 4. The nearly constant divergence of $\sim 30^{\circ} \mathrm{ob}-$ tained for the high ion temperatures is due to the, albeit 


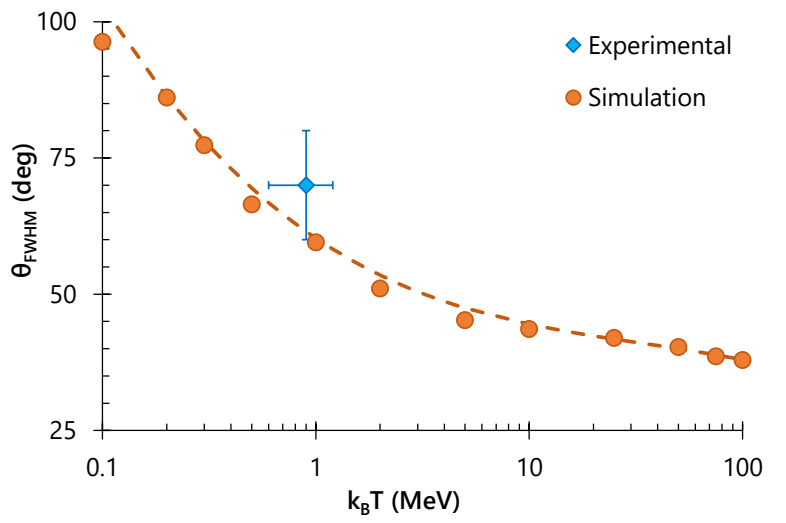

Figure 4: Neutron beam divergence (FWHM) as a function of the 288 ion beam temperature obtained from our simulations. The blue point 289 shows the beam divergence obtained for the $d(d, n)^{3}$ He reaction us- 290 ing the deuteron beam profile obtained from our experiment discussed 291 above. small, intrinsic divergence $\left(\sim 15^{\circ}\right)$ of the highest energy ions produced by the TNSA mechanism. This limita- 297 tion in the neutron beam divergence can be easily elim- ${ }^{298}$ inated by focussing the ion beams on the catcher, for ${ }_{300} 29$ example by using one of the several schemes reported ${ }_{301}$ in literature, such as permanent/pulsed magnetic focus- ${ }^{302}$ ing devices [23, 24, 25], laser-driven micro-lens[26], ${ }^{303}$ hemi-spherical targets [27], shaped targets [28] etc. Us- ${ }_{305}$ ing a focussed beam of ions with narrow energy band 306 can also help reducing the pulse duration of emitted fast ${ }^{307}$ neutrons, as recently reported by Higginson et al. [29]. ${ }_{300}^{300}$

\section{Conclusions}

We presented results obtained from a numerical ${ }^{31}$ model simulating the neutron beam generation by laser- ${ }_{320}$ driven ions in a pitcher-catcher scenario. Simulation re- ${ }^{221}$ sults are broadly consistent with the neutron beam pro- ${ }^{322}$ file observed in the experiment while using the experi- ${ }^{323}$ mentally measured ion beam profile in the simulation. ${ }_{325}$ By varying the ion beam parameters, simulations pre- 326 dict improvement in the neutron beam divergence with ${ }^{327}$ an increase in the ion beam temperature and cut-off en- ${ }_{329}{ }^{328}$ ergy, as expected from the TNSA mechanism at higher laser intensities. Further experimental measurements 331 with improved ion beam parameters would be required ${ }^{332}$ to benchmark the simulated trend for neutron beam di- ${ }_{334}^{333}$ vergence.

\section{Acknowledgements}

The authors acknowledge funding from EPSRC [EP/J002550/1-Career Acceleration Fellowship held by S. K., EP/L002221/1, EP/K022415/1, EP/J500094/1. Authors acknowledge support of engineering, target fabrication and experimental science groups of Central Laser Facility of STFC, UK.

[1] L. Gray, J. Read, Treatment of cancer with fast neutrons, Nature 152 (1943) 53

[2] A. Buffler, Contraband detection with fast neutrons, Radiation Physics and Chemistry 71 (3) (2004) 853-861.

[3] D. Vartsky, I. Mor, M. Goldberg, D. Bar, G. Feldman, V. Dangendorf, K. Tittelmeier, M. Weierganz, B. Bromberger, A. Breskin, Novel detectors for fast-neutron resonance radiography, Nuclear Instruments and Methods in Physics Research Section A: Accelerators, Spectrometers, Detectors and Associated Equipment 623 (1) (2010) 603-605.

[4] L. Perkins, B. Logan, M. Rosen, M. Perry, T. D. de la Rubia, N. Ghoniem, T. Ditmire, P. Springer, S. Wilks, The investigation of high intensity laser driven micro neutron sources for fusion materials research at high fluence, Nuclear Fusion 40 (1) (2000) 1.

[5] A. Wiedenmann, U. Keiderling, K. Habicht, M. Russina, R. Gähler, Dynamics of field-induced ordering in magnetic colloids studied by new time-resolved small-angle neutronscattering techniques, Physical review letters 97 (5) (2006) 057202.

[6] S. Kar, A. Green, H. Ahmed, A. Alejo, A. Robinson, M. Cerchez, R. Clarke, D. Doria, S. Dorkings, J. Fernandez, et al., Beamed neutron emission driven by laser accelerated light ions, New J. Phys., 18, 053002 (2016).

[7] A. Alejo, H. Ahmed, A. Green, S.R. Mirfayzi, M. Borghesi, S. Kar, Recent Advances in laser-driven neutron sources, Il Nuovo Cimento C (In press)

[8] S. Atzeni, J. Meyer-ter Vehn, The Physics of Inertial Fusion: BeamPlasma Interaction, Hydrodynamics, Hot Dense Matter: BeamPlasma Interaction, Hydrodynamics, Hot Dense Matter, Vol. 125, Oxford University Press, 2004.

[9] A. Macchi, M. Borghesi, M. Passoni, Reviews of Modern Physics 85 (2013) 751.

[10] Extreme light infraestracture(eli) (http://www.eli-np.ro/).

[11] J. Zou, C. Le Blanc, D. Papadopoulos, G. Chériaux, P. Georges, G. Mennerat, F. Druon, L. Lecherbourg, A. Pellegrina, P. Ramirez, et al., Design and current progress of the apollon 10 pw project, High Power Laser Science and Engineering 3 (2015) e2.

[12] J. Davis, G. Petrov, Angular distribution of neutrons from highintensity laser-target interactions, Plasma Physics and Controlled Fusion 50 (6) (2008) 065016.

[13] D. B. Pelowitz, et al., Mcnpx users manual version 2.5. 0, Los Alamos National Laboratory 76.

[14] D. Gwynne, S. Kar, D. Doria, H. Ahmed, M. Cerchez, J. Fernandez, R. Gray, J. Green, F. Hanton, D. MacLellan, P. McKenna, Z. Najmudin, D. Neely, J. Ruiz, A. Schiavi, M. Streeter, M. Swantusch, O. Willi, M. Zepf, M. Borghesi, Modified thomson spectrometer design for high energy, multispecies ion sources, Review of Scientific Instruments 85 (3). doi:10.1063/1.4866021.

[15] G. Petrov, D. Higginson, J. Davis, T. B. Petrova, J. McNaney, C. McGuffey, B. Qiao, F. Beg, Generation of high-energy (i 15 mev) neutrons using short pulse high intensity lasers, Physics of Plasmas (1994-present) 19 (9) (2012) 093106. 
[16] C. Danson, P. Brummitt, R. Clarke, J. Collier, B. Fell, A. Frack- 401 iewicz, S. Hancock, S. Hawkes, C. Hernandez-Gomez, P. Hol- 402 ligan, et al., Vulcan petawattan ultra-high-intensity interaction facility, Nuclear Fusion 44 (12) (2004) S239.

[17] A. Alejo, S. Kar, H. Ahmed, A. Krygier, D. Doria, R. Clarke, J. Fernandez, R. Freeman, J. Fuchs, A. Green, J. Green, D. Jung, A. Kleinschmidt, C. Lewis, J. Morrison, Z. Najmudin, H. Nakamura, G. Nersisyan, P. Norreys, M. Notley, M. Oliver, M. Roth, J. Ruiz, L. Vassura, M. Zepf, M. Borghesi, Characterisation of deuterium spectra from laser driven multi-species sources by employing differentially filtered image plate detectors in thomson spectrometers, Review of Scientific Instruments 85 (9). doi: $10.1063 / 1.4893780$

[18] F. Nürnberg, M. Schollmeier, E. Brambrink, A. Blažević, D. Carroll, K. Flippo, D. Gautier, M. Geißel, K. Harres, B. Hegelich, et al., Radiochromic film imaging spectroscopy of laser-accelerated proton beams, Review of scientific instruments 80 (3) (2009) 033301.

[19] P. Mora, Plasma expansion into a vacuum, Phys. Rev. Lett. 90 (2003) 185002. doi:10.1103/PhysRevLett.90.185002.

URL http://link.aps.org/doi/10.1103/ PhysRevLett.90.185002

[20] J. Fuchs, P. Antici, E. dHumières, E. Lefebvre, M. Borghesi, E. Brambrink, C. Cecchetti, M. Kaluza, V. Malka, M. Manclossi, et al., Laser-driven proton scaling laws and new paths towards energy increase, Nature Physics 2 (1) (2005) 48-54.

[21] L. Robson, P. Simpson, R. Clarke, K. Ledingham, F. Lindau, O. Lundh, T. McCanny, P. Mora, D. Neely, C.-G. Wahlström, et al., Scaling of proton acceleration driven by petawatt-laserplasma interactions, Nature Physics 3 (1) (2006) 58-62.

[22] A. Krygier, J. Morrison, S. Kar, H. Ahmed, A. Alejo, R. Clarke, J. Fuchs, A. Green, D. Jung, A. Kleinschmidt, et al., Selective deuterium ion acceleration using the vulcan petawatt laser, Physics of Plasmas (1994-present) 22 (5) (2015) 053102.

[23] M. Schollmeier, S. Becker, M. Geißel, K. Flippo, A. Blažević, S. Gaillard, D. Gautier, F. Grüner, K. Harres, M. Kimmel, et al., Controlled transport and focusing of laser-accelerated protons with miniature magnetic devices, Physical review letters 101 (5) (2008) 055004.

24] M. Nishiuchi, I. Daito, M. Ikegami, H. Daido, M. Mori, S. Orimo, K. Ogura, A. Sagisaka, A. Yogo, A. Pirozhkov, et al., Focusing and spectral enhancement of a repetition-rated, laser-driven, divergent multi-mev proton beam using permanent quadrupole magnets, Applied Physics Letters 94 (6) (2009) 061107.

[25] S. Ter-Avetisyan, M. Schnürer, R. Polster, P. Nickles, W. Sandner, First demonstration of collimation and monochromatisation of a laser accelerated proton burst, Laser and Particle Beams 26 (04) (2008) 637-642.

[26] T. Toncian, M. Borghesi, J. Fuchs, E. d'Humières, P. Antici, P. Audebert, E. Brambrink, C. A. Cecchetti, A. Pipahl, L. Romagnani, et al., Ultrafast laser-driven microlens to focus and energy-select mega-electron volt protons, Science 312 (5772) (2006) 410-413.

[27] S. Kar, K. Markey, M. Borghesi, D. Carroll, P. McKenna, D. Neely, M. Quinn, M. Zepf, Ballistic focusing of polyenergetic protons driven by petawatt laser pulses, Physical review letters 106 (22) (2011) 225003.

[28] S. Kar, K. Markey, P. Simpson, C. Bellei, J. Green, S. Nagel, S. Kneip, D. Carroll, B. Dromey, L. Willingale, et al., Dynamic control of laser-produced proton beams, Physical review letters 100 (10) (2008) 105004.

[29] D. Higginson, L. Vassura, M. Gugiu, P. Antici, M. Borghesi, S. Brauckmann, C. Diouf, A. Green, L. Palumbo, H. Petrascu, et al., Temporal narrowing of neutrons produced by high-intensity short-pulse lasers, Physical review letters 115 (5) (2015) 054802 